\title{
Studies on the Biosynthesis of Psoralen and Bergapten in the Leaves of Ficus carica
}

\author{
G. Caporale, F. Dall'Acqua, S. Marciani, and A. Capozzi
}

Istituto di Chimica Farmaceutica, Centro di Chimica del Farmaco e dei prodotti biologicamente attivi, University of Padua (Italy)

(Z. Naturforsch. 25 b, 700—703 [1970]; eingegangen am 11. März 1970)

\begin{abstract}
With the aim of studying the biosynthesis of psoralen and bergapten, two furocoumarins present in the leaves of "Ficus carica" (Moraceae), the Authors, continuing preceding researchs on this topic, have fed the leaves with the following labelled precursors: $4^{\prime}, 5^{\prime}$-dihydropsoralen, $4^{\prime}, 5^{\prime}$-dihydrobergapten, 7-hydroxycoumarin, 5,7-dihydroxycoumarin and 5-methoxy-7-hydroxycoumarin.

The results obtained indicate that all these substances are certain biogenetic precursors for psoralen and bergapten.

On the basis of the results obtained the biosynthetic pathway of furocoumarins seems to involve first of all the formation of a coumarinic derivative 7-hydroxylated, then an isoprenylation reaction which leads to the formation of the hydrogenated furan ring, finally dehydrogenation of the $4^{\prime}, 5^{\prime}$-dihydrofurocoumarins to psoralen and bergapten.
\end{abstract}

Psoralen (1) and bergapten (2) show, like other natural or synthetic furocoumarins, various interesting photobiological properties. The skin-photosensitizing activity of furocoumarins has been widely studied for several years in this Institute. Recently the mechanism of action of these substances, which involves a photoreaction with DNA, has been clarified ${ }^{1}$.

On the biosynthesis of furocoumarins only a few studies have been made, while a large number of investigations have been carried out on the biosynthesis of coumarin and of some of its derivatives ${ }^{2}$.

In the case of furocoumarins the following two pathways may be suggested:

1. biosynthesis in a first step of a benzofuran-derivative and then formation of the $\alpha$-pyrone ring;

2 . biosynthesis of a coumarin derivative and then formation of the furan ring.

In a previous research on the biosynthesis of psoralen (1) and of bergapten (2), contained in the leaves of "Ficus carica" (Moraceae), CAPORALE and coworkers ${ }^{3}$ had used various substances which could lead to the biosynthesis of the furocoumarins through both the above mentioned pathways 1 and 2 .

The precursors used were: tyrosine $-2 \cdot{ }^{14} \mathrm{C}$, tyrosine $-{ }^{3} \mathrm{H}$, mevalonic acid $-2 \cdot{ }^{14} \mathrm{C}$, acetate $-2 \cdot{ }^{3} \mathrm{H}$ and succinic acid $-2,3-{ }^{3} \mathrm{H}$. Mevalonic acid $\therefore$ as been effectively incorporated in the two furo-

${ }^{1}$ For a review of this topic see: L. Musajo and G. RoDighiero, Photochem. Photobiol. 11, 27 [1970].

2 For a review see: S. A. Brown, Biosynthesis of Aromatic Compounds, vol. 3, pag. 15, Pergamon Press, Oxford 1966.

3 G. Caporale, A. Breccia, and G. Rodighiero, Prepn. Bio-Med. Appl. Labeled Mol., Proc. Sympos., pag. 103, Venice 1964. coumarins, while the incorporation of the other substances occurred in a smaller amount.

Although from the experiments with mevalonic acid $-2-{ }^{14} \mathrm{C}$ it was not possible to establish the position of the ${ }^{14} \mathrm{C}$ in the molecule of the two labelled furocoumarins, we may consider that the two carbon atoms in the position $-4^{\prime}$ - and $-5^{\prime}$. of the furan ring, derive from this precursor; also in the case of the leaves of "Ficus carica" we believe to be valid what FLoss and Mothes ${ }^{4}$ and Floss and PAIKERT ${ }^{5}$ have demonstrated for the biosynthesis of furocoumarins in the roots of "Pimpinella magna", namely that mevalonic acid takes part in the biosynthesis of the furan ring.

The same researchers demonstrated that in this plant biosynthesis occurs as indicated in pathway 2 .

With the aim of studying more widely the biosynthesis of furocoumarins in fig-leaves and of ascertaining if also in this plant the pathway 2 is valid, we have performed some experiments using as precursors the following labelled substances: $4^{\prime}, 5^{\prime}$-dihydropsoralen (3), 4', $5^{\prime}$-dihydrobergapten (4), 7-hydroxycoumarin or umbelliferone (5), 5,7-dihydroxycoumarin (6) and 5-methoxy-7-hydroxycoumarin (7). The choice of these precursors had been in part suggested by a preceding work in which very small quantities of $4^{\prime}, 5^{\prime}$-dihydropsoralen (3) and 7-hydroxycoumarin (5), other than psoralen and bergapten, had been isolated from fig-leaves ${ }^{6}$.

${ }^{4}$ H. G. Floss and U. Mothes, Phytochem. 5, 161 [1966].

5 H. G. Floss and H. Paikert, Phytochem. 8, 589 [1969].

6 F. Dall'Acqua, S. Marciani, and G. Chiarelotto, Atti Ist. Veneto Sci. Lettere Arti, Cl. Sci. mat. natur. 126, 103 [1968]. 

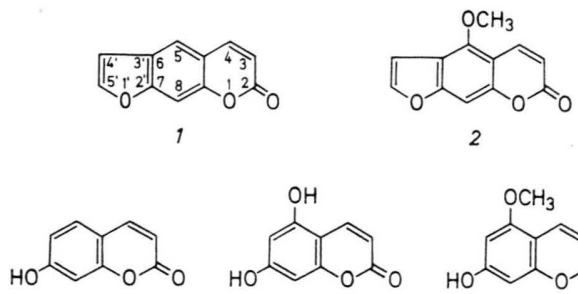<smiles>O=c1ccc2c(O)cc(O)cc2o1</smiles>

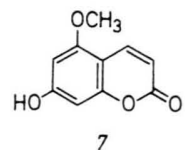

5

6

\section{Materials and Methods}

\section{a) Preparation of precursors}

$4^{\prime}, 5^{\prime}$-dihydropsoralen ${ }^{7}, 4^{\prime}, 5^{\prime}$-dihydrobergapten ${ }^{8}, 7$-hydroxycoumarin ${ }^{9}, 5,7$-dihydroxycoumarin ${ }^{10}$ and 5-methoxy-7-hydroxycoumarin ${ }^{11}$ have been prepared by synthesis and then tritiated by the WILzBACH method ${ }^{12}$.

$4^{\prime}, 5^{\prime}$-dihydropsoralen (3) and $4^{\prime}, 5^{\prime}$-dihydrobergapten (4) have been tritiated according to the method described elsewhere ${ }^{13}$. The other substances [7-hydroxycoumarin (5), 5,7-dihydroxycoumarin (6) and 5-methoxy-7-hydroxycoumarin (7)] after an extended contact with tritium ( 2 months) in sealed glass tubes were purified as indicated in the following steps: dissolution in $\mathrm{NaOH} 2.5 \mathrm{~N}$ and precipitation with $\mathrm{HCl} 2.7 \mathrm{~N}$; crystallization from water (5 and 6) and from water: ethyl alcohol [8:2(v/v)] (7); sublimation in high vacuum and crystallization from water-ethyl alcohol (6:4; $\mathrm{v} / \mathrm{v})$; - chromatography on silica gel thin-layer preparative chromatoplates (Merck cat. 5717); solvent: ethyl acetate-cyclohexane $(3: 1 ; \mathrm{v} / \mathrm{v})$; elution with absolute ethyl alcohol and filtration through a Millipore Mitef $5 \mu$ filter. The U.V. spectra of the labelled substances were identical with those of pure compounds.
The radiochemical purity of these substances was tested by thin layer chromatography on analytical silica gel chromatoplates (Merck cat. 5715) using ethyl acetatecyclohexane $(3: 1 ; \mathrm{v} / \mathrm{v})$ as solvent of development. The specific activities of the labelled precursors are indicated in Table II.

\section{b) Feeding fig-leaves with labelled precursors}

The stems of freshly cut fig-leaves were introduced into test tubes containing the saturated aqueous solution of the precursor ( $2 \mathrm{ml}$ of solution for every leaf). The metabolism time lasted 72 hours: during this period distilled water was added to the test tubes and the room was illuminated with two $500 \mathrm{~W}$ Osram HWL lamps.

Check experiments performed under the same conditions with labelled precursors exclude a spontaneous transformation of $4^{\prime}, 5^{\prime}$-dihydropsoralen and $4^{\prime}, 5^{\prime}$-dihydrobergapten in the corresponding furocoumarins.

Extraction of the furocoumarins

After the metabolism time the leaves treated with the same precursor were collected, dried, cut in minute pieces and then extracted with methyl alcohol in an extraction apparatus; the methanolic extract after concentration to a volume of $150 \mathrm{ml}$ was added of an equal volume of aqueous $\mathrm{KOH}(10 \%)$. After two hours the alkaline alcoholic solution was extracted with ether and then acidified with $\mathrm{HCl}$. After twelve hours, the acidic solution was thoroughly extracted with ether; the etheral extract was then washed with a saturated $\mathrm{NaHCO}_{3}$ solution and dried over $\mathrm{Na}_{2} \mathrm{SO}_{4}$. After distillation of the solvent, the residue was sublimated under vacuum $\left[0.001 \mathrm{~mm}\right.$ up to $200^{\circ}$ (the temperature of the air bath)] and then the sublimate was dissolved in methanol to become the "coumarinic extract" 3,14 .

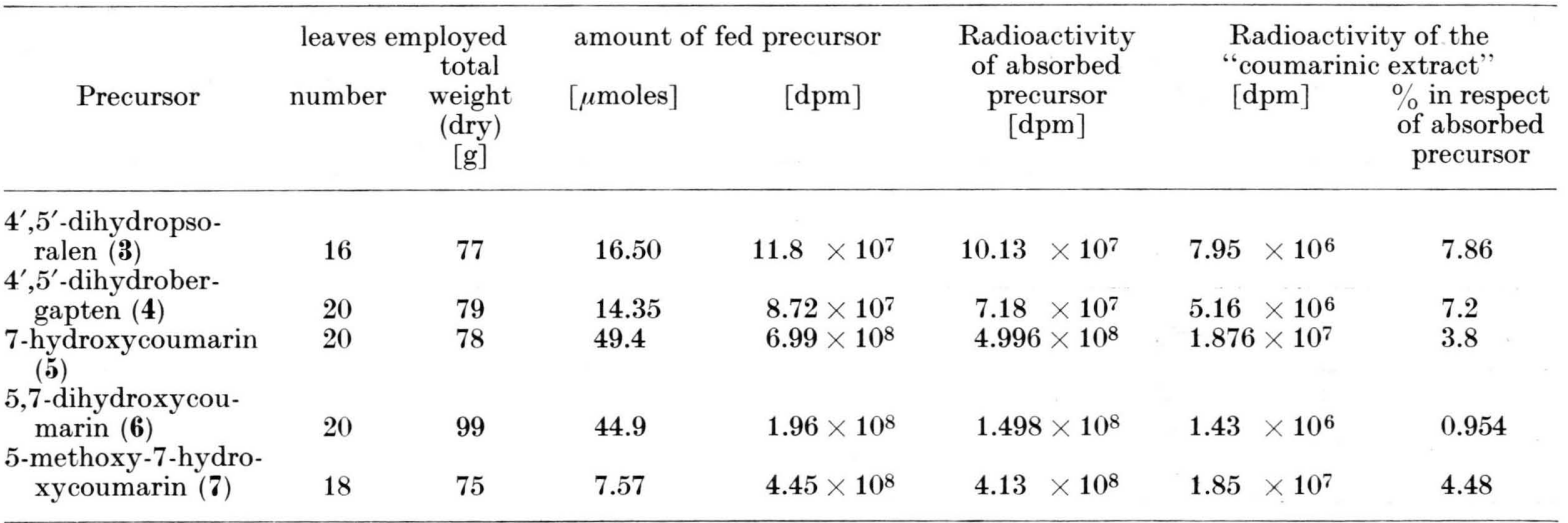

Table I. Incorporation of precursors into "coumarinic extract".

7 E. C. Horning and D. B. Reisner, J. Amer. chem. Soc. 70, 3619 [1948].

8 G. Caporale, Ann. Chimica 48, 650 [1958].

9 H. V. Pechmann, Chem. Ber. 17, 929 [1884].

10 R. G. Heyes and A. Robertson, J. chem. Soc. [London] 1936, 1831 .
11 G. Rodighiero and C. Antonello, Farmaco [Pavia] 10, 889 [1955].

12 K. E. Wilzbach, J. Amer. chem. Soc. 79, 1013 [1957].

13 S. Marciani, F. Dall'Acqua, and C. Colombini, Ann. Chimica 59, 1067 [1969].

14 E. SpÄTH, Chem. Ber. 70 A, 83 [1937]. 
Separation of psoralen and bergapten

A first stage of the separation was carried out with alumina preparative thin-layer chromatoplates; (Merck, cat. 5726); $2 \mathrm{ml}$ of the "coumarinic extract" solution were placed on every chromatoplate in bands and chloroform was used as solvent of development; in this step the traces of precursors which should be mixed with the furocoumarins were removed. Bergapten and psoralen showed the same $R_{f}=0.72$, the precursors had different $R_{f}$ values, that is: $4^{\prime}, 5^{\prime}$-dihydropsoralen $R_{f}=0.63,4^{\prime}, 5^{\prime}$-dihydrobergapten $R_{f}=0.56 ; 7$-hydroxycoumarin, 5,7-dihydroxycoumarin and 5-methoxy-7-hydroxycoumarin remained in the place where the band was initially deposed.

The areas corresponding to psoralen and bergapten were eluted with absolute ethyl alcohol and the solutions so obtained, after concentration, were placed in bands on chromatopapers Whatman $3 \mathrm{MM}$; the chromatograms were developed with water and then dried. The areas corresponding to psoralen and bergapten, identified by their different fluorescence when observed at $365 \mathrm{~nm}$ light (blue-grey for psoralen and yellowgreen for bergapten) were eluted separately with ethyl alcohol, concentrated and then newly placed in bands on chromatopapers and developed under the same conditions. The solutions obtained by the elution respectively of psoralen and bergapten with ethyl alcohol showed U.V. spectra identical to those of pure compounds; the concentration of psoralen was determined by spectrophotometric reading at $332 \mathrm{~nm}(\varepsilon=7730){ }^{15}$ and that of bergapten at $310 \mathrm{~nm}(\varepsilon=15140){ }^{15}$.

The radiochemical purity of psoralen and bergapten was tested by thin layer chromatography; silica gel analytical chromatoplates (Merck, cat. 5715) were used; solvent ethyl acetate-cyclohexane $(1: 2 ; \mathrm{v} / \mathrm{v})^{16}$. Radiochemical measurements were performed by means of a Beckman LS 150 liquid scintillation spectrometer, using a dioxane-base scintillator * (the apparatus efficiency was $40 \%$ for tritium).

\section{Results and Discussion}

The results obtained are reported in Table II. Feeding fig-leaves with $4^{\prime}, 5^{\prime}$-dihydropsoralen (3) leads to the formation essentially of labelled psoralen and only of a small amount of labelled bergapten, while feeding with $4^{\prime}, 5^{\prime}$-dihydrobergapten (4) leads to the labelling essentially of bergapten and only in a little extent of psoralen. Therefore the two dihydroderivatives are valuable precursors: they undergo a dehydrogenation reaction giving the corresponding furocoumarins. However the presence of a small amount of labelled bergapten in the first case and of labelled psoralen in the second one suggests that in addition to the principal dehydrogenation reaction other secondary reactions respectively of methoxylation (bergapten) and demethoxylation (psoralen) can occur.

The data obtained seem indicate that $4^{\prime}, 5^{\prime}$-dihydrobergapten forms bergapten more effectively than $4^{\prime}, 5^{\prime}$-dihydropsoralen forms psoralen: in fact dilution values are respectively 150 and 447 . In this respect however we point out that in fig-leaves psoralen is rather constantly present in an amount three times as great as bergapten ${ }^{17}$ : this fact may have significance to explain the different dilution values for the two furocoumarins.

Feeding fig-leaves with 7-hydroxycoumarin (5), 5,7-dihydroxycoumarin (6) and 5-methoxy-7-hydroxycoumarin (7) leads to the formation both of labelled psoralen and of labelled bergapten, even if in different amounts. In the psoralen molecule the effectiveness of the incorporation shows this order: 7-hydroxycoumarin (5) > 5,7-dihydroxycoumarin

\begin{tabular}{|c|c|c|c|c|c|}
\hline Precursor & $\begin{array}{c}\text { specific activity } \\
\text { of precursor } \\
{\left[\mathrm{dpm} / \mu \text { moles } \times 10^{6}\right]}\end{array}$ & $\begin{array}{l}\text { specific activity } \\
\text { of psoralen } \\
{[\mathrm{dpm} / \mu \text { moles }]}\end{array}$ & $\begin{array}{l}\text { dilution * } \\
\text { of } \\
\text { psoralen }\end{array}$ & $\begin{array}{l}\text { specific activity } \\
\text { of bergapten } \\
{[\mathrm{dpm} / \mu \text { moles }]}\end{array}$ & $\begin{array}{l}\text { dilution * } \\
\text { of } \\
\text { bergapten }\end{array}$ \\
\hline $4^{\prime}, 5^{\prime}$-dihydropsoralen & 7.15 & $1.6 \times 10^{4}$ & 447 & $1.222 \times 10^{3}$ & 5860 \\
\hline $4^{\prime}, 5^{\prime}$-dihydrobergapten & 6.08 & $0.64 \times 10^{3}$ & 9500 & $4.06 \times 10^{4}$ & 150 \\
\hline 7-hydroxycoumarin & 14.15 & $3.305 \times 10^{3}$ & 4280 & $1.585 \times 10^{3}$ & 8920 \\
\hline 5,7-dihydroxycoumarin & 4.37 & $0.822 \times 10^{3}$ & 5320 & $0.549 \times 10^{3}$ & 7980 \\
\hline $\begin{array}{l}\text { 5-methoxy-7-hydroxy- } \\
\text { coumarin }\end{array}$ & 58.8 & $8.4 \times 10^{3}$ & 7000 & $11.48 \times 10^{3}$ & 5120 \\
\hline
\end{tabular}

Table II. Specific activity of precursors and of biosynthesized furocoumarins and relative dilutions. * Dilution is defined as the ratio between the specific activity of the precursor and that of the extracted furocoumarin.

* $120 \mathrm{~g}$ naphthalene, 2,5-diphenyl-oxazole g 4, 2,2'-p-phenylen-bis (5-phenyl-oxazole) g 0,075 in dioxane up to $1000 \mathrm{ml}$ of solution.

15 G. Rodighiero and V. Cappellina, Gazz. chim. ital. 91, 103 [1961].
16 F. Dall'Acqua, S. Marciani, and G. Rodighiero, Z. Naturforsch. 24 b, 307 [1969].

17 G. Rodighiero and C. Antonello, Farmaco [Pavia] 14, 679 [1959]. 
(6) >5-methoxy-7-hydroxycoumarin (7). By contrary in the bergapten molecule the order of incorporation is inverted.

We may conclude that these three coumarin derivatives are effective precursors, even if incorporation is lower than that found for the two $4^{\prime}, 5^{\prime}$-dihydrofurocoumarins. This seems to support the possibility of biosynthesis of the furan ring on a coumarin derivative; as it was indicate by FLoss et al. ${ }^{4,5}$, this fact should involve an isoprenylation reaction.

Considering all the results obtained we may assume that the biosynthesis of furocoumarins in figleaves may occur as indicated in the above reported a)

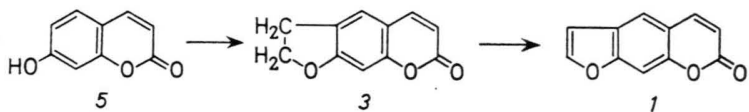

b)<smiles>C=C1C=Cc2c(OC)cc(O)cc2O1</smiles><smiles>COc1c2c(cc3oc(=O)ccc13)OCC2</smiles><smiles>COc1c2ccoc2cc2oc(=O)ccc12</smiles>

pathway N.2, that is in a first step there would take place the biosynthesis of the coumarin moiety, then the formation of the hydrogenated furan ring and lastly a dehydrogenation reaction with the formation of psoralen and bergapten.

Moreover the results indicate that there exists a clear specificity of 7-hydroxycoumarin (5) for the biosynthesis of psoralen and of 5-methoxy-7-hydroxycoumarin (7) for that of bergapten. It is however interesting to observe that the biosynthetic schemes indicated as a and b in Fig. 2 are not independent one from the other, but that there exists the possibility of intercrossing, by means of methoxylation or demethoxylation reactions. Both furocoumarins should so derive from one single precursor only and this may be 7-hydroxycoumarin (5) (umbelliferone) which is present, even if in a very small amount, in the fig-leaves ${ }^{6}$.

We are indebted to Prof. Luigi Musajo and Prof. GiovanNi RODIGHIERo for helpful discussion on this research. 